\title{
Operative Versorgung von Sprunggelenksfrakturen - ein Anfängereingriff
}

\author{
Rupert Ketterl
}

\section{Einleitung}

Die exakte Führung des Talus in der Sprunggelenksgabel ist die Voraussetzung für eine ungestörte Funktion des oberen Sprunggelenks. Abweichungen führen unweigerlich $\mathrm{zu}$ unphysiologischen Belastungen und damit zu einer Inkongruenzarthrose [1,2]. Knöcherne und ligamentäre Verletzungen dieser Region wurden systematisch von Weber [4] und von Lauge-Hansen [2] beschrieben. Die operative Versorgung umfasst dabei das Ziel einer stufenlosen Rekonstruktion der Gelenkflächen und eine stabile/elastische Führung der Gelenkgabel. Folgende Voraussetzungen sind dabei zu berücksichtigen:

- Exakte Wiederherstellung der Fibula unter Beachtung von Länge, Rotation, Achse und Stellung in der Incisur

- Einpassung des Innenknöchels

- Anatomische Rekonstruktion zusätzlicher Tibiafragmente

- Versorgung instabiler Syndesmosenverletzungen und Bandrupturen

Häufig wird die Schwere der vorliegenden Sprunggelenksverletzungen unterschätzt. Operative Eingriffe in dieser Region werden als Anfängereingriffe eingeordnet. Das vorgestellte Beispiel sollte auf die Komplexität von Verletzungen im Sprunggelenksbereich und die daraus resultierende Therapierelevanz hinweisen.

\section{Fallvorstellung}

Ein 64-jähriger Patient erleidet im Rahmen eines Fahrradunfalls eine Sprunggelenksluxationsfraktur, $1^{\circ}$-ig offen, mit einer Weichteilläsion im Bereich der distalen Tibia medial. Am Unfallort wird eine Reposition der Fraktur durchgeführt. Die Unfallaufnahmen zeigen eine Fibulafraktur, eine Fraktur des Innen-

OP-JOURNAL 2008; 24: 254-255

(c) Georg Thieme Verlag KG Stuttgart • New York DOI 10.1055/s-2008-1039019 knöchels sowie ein großes hinteres Volkmann-Dreieck (Abb.1).

Aufgrund der Weichteilsituation erfolgt am Unfalltag die Anlage eines gelenküberbrückenden Fixateur externe, wobei die distale Schanz'sche Schraube im Bereich der Tibia in das Volkmann-Dreieck platziert wurde (Abb. 2 c). Zur Festlegung der Verletzungsschwere erfolgte die Durchführung einer Computertomografie. Hier zeigen die axialen Aufnahmen eine Beteiligung des Innenknöchels sowie ein großes hinteres VolkmannDreieck. Die Fibula ist bis zur Fibulaspitze längs gespalten (Abb.2a). Die koronaren Schichten zeigen das große Innenknöchelfragment sowie die langstreckige Ausdehnung der Verletzung im Bereich der distalen Fibula (Abb. 2 b). In der seitlichen Rekonstruktion wird neben der Verletzung der Fibula die Ausdehnung des hinteren VolkmannDreiecks dokumentiert (Abb. 2 c).

Nach Weichteilkonsolidierung erfolgt am 4. posttraumatischen Tag die interne Osteosynthese mit 8-Loch-Drittelrohrplatte und Einbringen von 2 interfragmentären Zugschrauben im Bereich der Fibula. Die Innenknöchelfraktur wird über einen limitierten Zugang mittels 3er-Zugschrauben stabilisiert. Das hintere Volkmann-Dreieck wird mit 2 Zugschrauben indirekt stabilisiert (Abb. 3).

Es gelingt eine stufenfreie Rekonstruktion der Gelenkfläche. Auf eine Stellschraube konnte verzichtet werden, nachdem die dynamische Untersuchung nach Komplettierung der Osteosynthese stabile Verhältnisse zeigte.

Der Heilverlauf war komplikationslos. Der Patient wurde mit einer dorsalen Kunststoffschiene bis zum Abschluss der Wundheilung ruhiggestellt. Dann erfolgte nach Anlage eines VacopedStiefels die schrittweise Belastungssteigerung, die nach 6 Wochen erlaubt war. Im weiteren Verlauf klagte der Patient

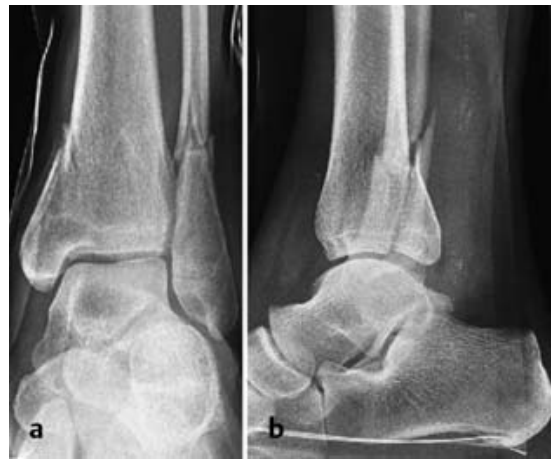

Abb. 1 a und b Unfallbild a.-p. (a) und seitlich (b) nach Reposition der Luxation durch den Rettungsdienst.
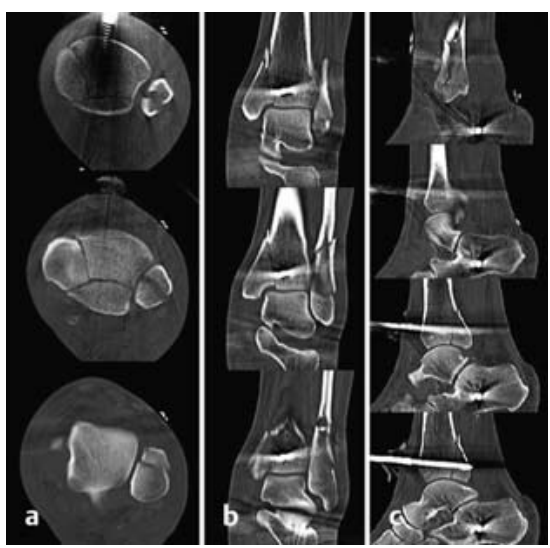

Abb. 2a bis c Das Ausmaß der Verletzung an Tibia und Fibula sind dargestellt. a Axiale Schichten der Computertomografie. b Koronare Schichten der Computertomografie. c Seitliche Rekonstruktion im CT.

über belastungsabhängige Schmerzen. Es zeigte sich 4 Monate nach operativer Versorgung eine zunehmende Schmerzhaftigkeit. Die radiologische Kontrolle ergab nun eine verzögerte Bruchheilung mit Auftreten einer Varusfehlstellung sowie der Entwicklung einer Rekurvation. Es war eine zusätzliche Fraktur im Bereich der distalen Tibia ventral zu erkennen (Abb. 4).

Die CT-Diagnostik zeigte in den axialen Schichten eine fehlende knöcherne 


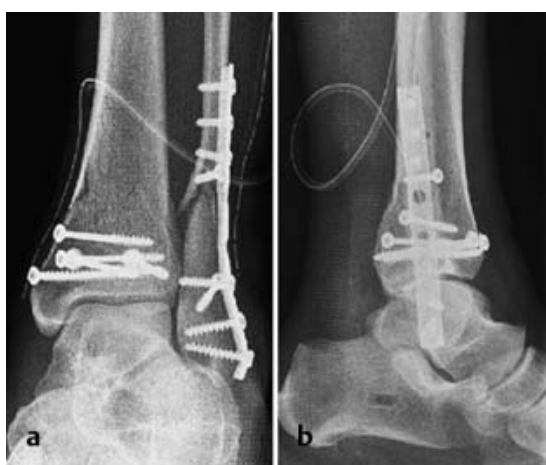

Abb. $\mathbf{3} \mathbf{a}$ und $\mathbf{b}$ a Postoperatives Bild nach osteosynthetischer Versorgung der Fibula mittels Drittelrohrplatte und interfragmentären Zugschrauben sowie Versorgung der Tibia mittels 3er-Zugschrauben im Innenknöchelbereich und 2er-Zugschrauben für das hintere Volkmann-Dreieck. b Postoperatives Bild seitlich.
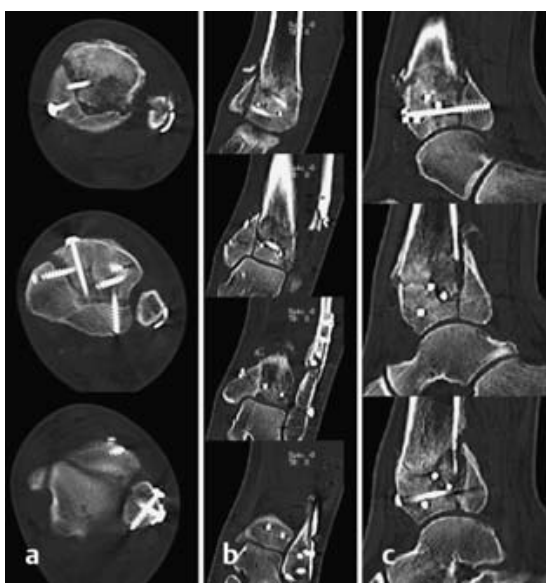

Abb. $\mathbf{5 a}$ bis c CT 4 Monate nach Osteosynthese. Axiale Schichten (a), koronare Schichten (b), seitliche Rekonstruktion (c).

Überbrückung sowohl des VolkmannDreiecks als auch des Innenknöchelfragments. Die Lokalisation der interfragmentären Zugschrauben für das Volkmann-Dreieck zeigt sich regelrecht (Abb.5a). In den koronaren Schichten in der Computertomografie lässt sich das Ausmaß der Varusfehlstellung sowie die fehlende knöcherne Überbrückung im Bereich der Fibula und der Tibia nachweisen (Abb.5b). Die seitliche Rekonstruktion zeigt die neu entstandene Fraktur im Bereich der Tibiavorderkante sowie das nicht knöchern fixierte hintere Volkmann-Dreieck trotz regelrechter Lage der von ventral her eingebrachten Zugschraube (Abb. 5c).

Bei dem Patienten war eine Korrekturoperation mit Aufhebung der Fehlstellung, Reosteosynthese im Bereich der Fibula mittels Drittelrohrplatte und im

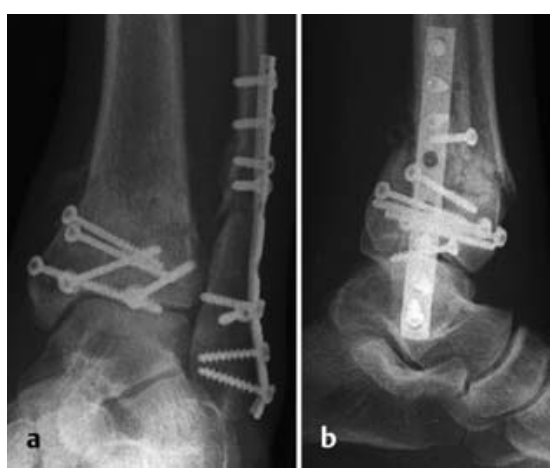

Abb. 4a und b Vier Monate nach operativer Versorgung. a Varusfehlstellung an der Fibula und an der Tibia bei verzögerter Bruchheilung. b Verzögerte Bruchheilung mit Rekurvation des distalen Tibiafragments mit zusätzlicher Ausbildung einer Fraktur an der distalen Tibia ventralseitig.

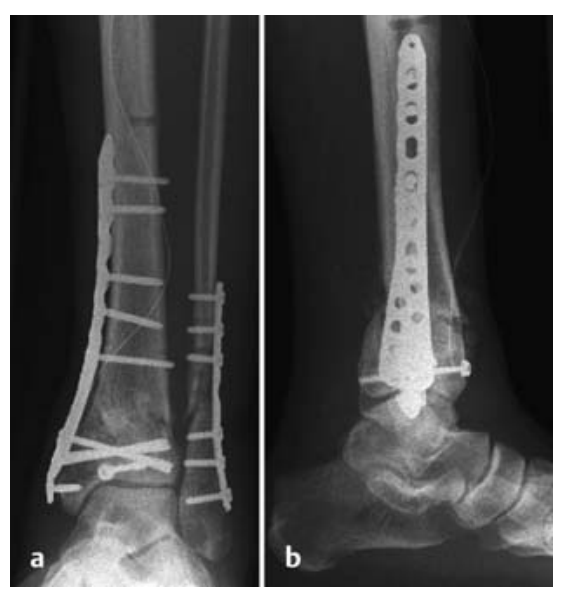

Abb. 6 a und b Postoperatives Bild nach Korrektur mit Reosteosynthese Fibula und Tibia, Spongiosaplastik und Anlage von Knochenwachstumsfaktoren.

Bereich der Tibia mittels winkelstabiler distaler Tibiaplatte in Kombination mit Spongiosaplastik und Anlage von Knochenwachstumsfaktoren (BMP 2) erforderlich (Abb. 6).

Bei dem Patienten zeigt sich nun ein regelrechter Verlauf nach Korrekturoperation. Er ist bei zugelassener Vollbelastung schmerzfrei mobilisiert.

\section{Diskussion}

Die Versorgung von Sprunggelenksverletzungen bedarf einer exakten Frakturanalyse unter Einbeziehung der Weichteilverhältnisse und Begleitverletzungen. Der Unfallhergang muss eruiert werden. Ziel der Versorgung ist eine stabile Osteosynthese, die eine funktionelle Nachbehandlung erlaubt.
Im vorgestellten Fall wurde nach möglichen Fehlern in der Behandlung gesucht. Dazu stellten wir uns folgende Fragen:

- Ausreichende Primärdiagnostik?

- Richtiger Operationszeitpunkt?

- Richtiges operatives Vorgehen?

- Richtige Implantatwahl?

- Richtige Nachbehandlung?

Die Erstbehandlung in unserem Fallbeispiel muss als korrekt bezeichnet werden. Die primäre Stabilisierung mittels Fixateur externe war bei $1^{\circ}$-ig offener Fraktur mit begleitender deutlicher Weichteilschwellung indiziert.

Das Ausmaß der knöchernen Verletzung wurde mittels CT geklärt. Nach erreichter Weichteilkonsolidierung erfolgte die definitive interne Versorgung verletzungsorientiert. Eine Unterschätzung der Traumatisierung der knöchernen Strukturen ist anhand des weiteren Verlaufes anzunehmen. Es ist bei der vorliegenden Verletzung der teils fließende Übergang einer Sprunggelenksverletzung zu einer Pilon-tibiale-Fraktur eher in Richtung der zuletzt genannten Verletzung gegeben. Eine primär stabile Osteosynthese im Bereich der distalen Tibia in Form einer Abstützplatte hätte aller Voraussicht nach das Ergebnis günstiger beeinflusst. Hinsichtlich der Nachbehandlung wurde der Forderung nach einer frühzeitigen funktionellen Therapie Rechnung getragen. Eine schrittweise Aufbelastung bei angelegtem Vacoped-Stiefel erfolgte anhand der radiologisch erreichten anatomischen Rekonstruktion des Sprunggelenkbereiches.

Fazit

Für die Behandlung von Frakturen im Sprunggelenksbereich ist Folgendes zu fordern:

Exakte Frakturanalyse - Berücksichtigung des Unfallmechanismus - Respektierung der Weichteilverhältnisse - Erreichen einer stabilen Osteosynthese - Verletzungsadaptierte frühzeitige Mobilisation mit funktioneller Nachbehandlung

\section{Literatur beim Verfasser}

\section{Prof. Dr. med. Rupert Ketterl}

Ärztlicher Direktor

Unfallchirurgie und Orthopädische Chirurgie, Zentrum Hand- und Wirbelsäulenchirurgie, Klinikum Traunstein, Cuno-Niggl-Straße 3, 83278 Traunstein

E-Mail:

rupert.ketterl@klinikum-traunstein.de 